\title{
Numerical Investigation of Combustion Instabilities in a Rocket Combustion Chamber with Supercritical Injection Using a Hybrid RANS/LES Method
}

\author{
Ansgar Lechtenberg* and Peter Gerlinger ${ }^{\dagger}$ \\ Institute of Combustion Technology, German Aerospace Center (DLR), Stuttgart, 70569, \\ Germany
}

\begin{abstract}
An experimentally measured model rocket combustion chamber is investigated numerically using a time resolving high order hybrid RANS/LES method. The selected operation point is characterized by weak combustion instabilities which are challenging to predict. The aim of this work is to enhance the prediction capabilities of combustion chamber instabilities by numerical methods. This aims on a better understanding of the reasons for combustion instabilities as well as on techniques to avoid them. The weak oscillating combustion instabilities found in experiment and simulation are analyzed in detail.
\end{abstract}

\section{Introduction}

Since the beginning of rocket development, combustion instabilities are an important subject of investigations. First discovered in the late 1930s, unstable oscillations were found in many, if not all rocket combustion chambers. ${ }^{1}$ They involve extreme loads in both low and high frequencies. The loads can be so severe that they cause a complete failure of the combustion chamber. This is especially critical for manned missions. To avoid this, the F-1 engine of the Saturn V rocket for example was tested over 2000 times in full-scale to solve problems with combustion instabilities. ${ }^{1}$ This causes enormous costs in the process of design.

Experimental investigations of rocket combustion chambers are often challenging due to extreme flow conditions. The propellants are injected cryogenically and/or at very high pressure. Additionally, the combustion products can reach temperatures up to roughly $4000 \mathrm{~K}$. Diagnostic possibilities in rocket combustion chambers are still limited due to these extreme conditions. Even in simplified model combustors, dynamic and static combustion chamber pressures, static wall temperatures and heat flues are often the only values measured quantitatively. Dynamic temperature measurements are often inaccurate due to the high response time of the sensors. In some cases it is also possible to install quartz windows in model rocket combustors to allow optical access. This makes capturing of shadowgraphs and $\mathrm{OH}^{*}$-chemiluminescence pictures possible. Quantitative optical diagnostics like laser-based flow field measurements are still impossible in most rocket combustion chamber flows.

Numerical simulations can support the design process of combustion chambers in combination with experiments. Good validated Computational Fluid Dynamics (CFD) codes can improve the understanding of the flow field behaviour in connection with experimental data. The full knowledge of the flow field enables analyses of the causes of combustion chamber instabilities, which can be verified in specially designed experiments. Up to now it is impossible to perform Direct Numerical Simulations (DNS) for rocket combustion chambers even for simple geometries as the one presented in this paper. The fine grids needed for DNS to resolve the fine turbulent scales in combination with the small timescales of combustion would require too much computing resources. To minimize the computational effort, the small scale turbulence can be modeled, while only the large scales are resolved. This is the concept of the Large-Eddy Simulation (LES).

*PhD Student, German Aerospace Center (DLR), Stuttgart, 70569, Germany

$\dagger$ Professor, German Aerospace Center (DLR), Stuttgart, 70569, Germany 
However, especially close to solid walls a good resolved LES requires still extremely fine grids in each coordinate direction to resolve the turbulence in the boundary layer accurately. On the other hand, impact of the near wall region on the main flow can be predicted properly by modern Reynolds-Averaged Navier-Stokes (RANS) turbulence models at relatively low costs. Therefore, to further reduce the computational effort, hybrid RANS/LES methods were proposed, where the near wall region is simulated by RANS and the main flow by LES. This principle is used in the in-house code Turbulent All Speed Combusiont Multigrid Solver $3 \mathrm{D}^{2}$ (TASCOM3D) to simulate combustion instabilities in rocket combustion chambers.

In the present paper, the experimentally investigated single injector rocket combustion chamber $\mathrm{BKC}^{3,4}$ is studied numerically. The chosen operation point $(\mathrm{OP})$ is characterised by weak oscillations and is described in detail in Sec. II. In former investigations, the occurring instabilities could not be reproduced by inexpensive 2D-Unsteady Reynolds-Averaged Navier-Stokes (URANS) simulations. ${ }^{5}$ Hence, the same operation point is simulated again using a hybrid RANS/LES method called improved Delayed Detached Eddy Simulation (iDDES) which is described in detail in Sec. III. The results are presented and discussed in Sec. IV.

\section{Test case description}

The investigated single injector combustion chamber BKC has been built and experimentally investigated at DLR Lampoldshausen. ${ }^{3,4}$ It has been designed to map the operating point of real upper stage engines. The combustion chamber is of cylindric shape and is completed by a convergent-divergent nozzle. Fig. 1 shows the axisymmetric BKC in a two dimensional plane. Furthermore geometric details are given in Table 1.

The combustion chamber can be operated with different fuel-oxidizer mixtures like hydrogen with oxygen or methane with oxygen. The mean combustion chamber pressure can be varied over a wide range from subto supercritical with respect to the critical pressure of oxygen. The injection temperature can be chosen from cryogenic to ambient. The test case discussed in this paper is a combustion of $\mathrm{H}_{2}$ and $\mathrm{O}_{2}$ at supercritical pressure ( 60 bar) with cryogenic injection (injection temperature is $\sim 120 \mathrm{~K}$ ). Thus, the conditions are similar to the operation point of the Vinci upper stage engine of the Ariane 6 rocket. ${ }^{6}$ A detailed description of the operation point is also given in Table 1.

Through the coaxial injector, $\mathrm{O}_{2}$ and $\mathrm{H}_{2}$ are injected separately. Mixing takes places directly downstream of the face plate where the diffusion flame is anchored. Shadow images have been captured through an optical access at the injector region. The dimension of the window is $100 \mathrm{~mm} \times 25 \mathrm{~mm}$ (location: see Fig. 1). To minimize the thermal loads on the window, a $\mathrm{H}_{2}$ cooling film is injected directly at the combustion chamber wall. Pressure sensors map the combustion chamber pressure during the firing test. Two monitor points (MP1 and MP2) at which the time signal of the pressure is analyzed in the simulation are also shown in Fig. 1.

As already mentioned, the chosen operation point exhibits weak oscillations only. The amplitude of the pressure oscillation is clearly below $1 \%$. This makes an accurate prediction of the combustion instabilities challenging as these oscillations may be damped by numerical dissipation.

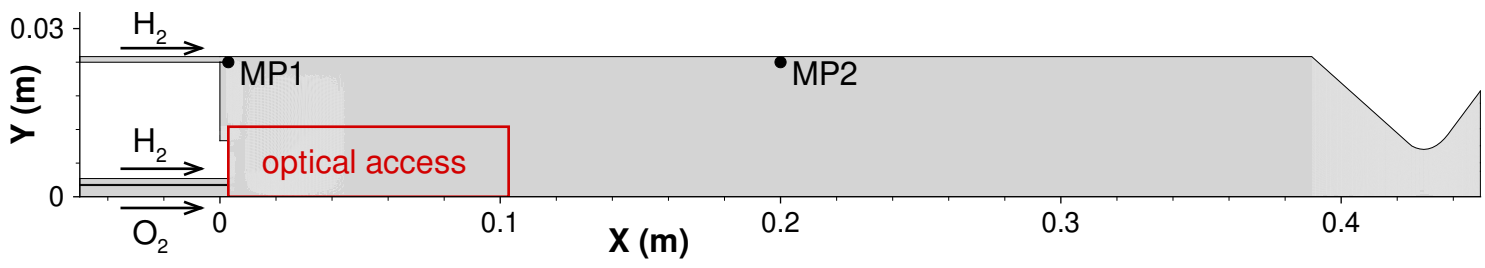

Figure 1. Geometry of the axisymmetric combustion chamber scaled in $x$-direction by a factor of two. MP1 and MP2 indicate the locations of monitor points. 
Table 1. Geometrical and operative details.

\begin{tabular}{lc}
\hline Geometrical details (specified in mm) & \\
\hline Number of injectors & 1 \\
Length of the combustion chamber & 430 \\
Diameter of the combustion chamber & 50 \\
Diameter of the nozzle throat & 16.8 \\
Diameter of the $\mathrm{O}_{2}$-injector & 4.0 \\
Inner diameter of the $\mathrm{H}_{2}$-injector & 4.4 \\
Outer diameter of the $\mathrm{H}_{2}$-injector & 6.5 \\
Height of the cooling film & 1.0 \\
\hline Operative details & \\
\hline Combustion chamber pressure $(\mathrm{bar})$ & 59,4 \\
Oxidizer to fuel ratio $($ injector $)$ & 4.83 \\
Global oxidizer to fuel ratio & 1.00 \\
Injection temperature $\mathrm{O}_{2}(\mathrm{~K})$ & 115.5 \\
Injection temperature $\mathrm{H}_{2}$, injector $(\mathrm{K})$ & 117.0 \\
Injection temperature $\mathrm{H}_{2}$, cooling film $(\mathrm{K})$ & 320.5 \\
Mass flow rate $\mathrm{O}_{2}(\mathrm{~kg} / \mathrm{s})$ & 0.29 \\
Mass flow rate $\mathrm{H}_{2}$, injector $(\mathrm{kg} / \mathrm{s})$ & 0.06 \\
Mass flow rate $\mathrm{H}_{2}$, cooling film $(\mathrm{kg} / \mathrm{s})$ & 0.23 \\
\hline
\end{tabular}

\section{Numerical method}

All simulation are performed with the in-house code TASCOM3D. TASCOM3D solves the full set of the three-dimensional compressible Navier Stokes equations containing conservation equation of mass, momentum and energy. Additionally for combustion, the conservation equations of a number of species are solved.

For the described test case, former investigations have shown, that numerical methods with low computational effort like 2D-URANS simulations did not resolve the weak oscillations. ${ }^{5}$ Therefore, a high order hybrid RANS/LES method (iDDES) is used. The near wall region is simulated by URANS using the k- $\omega-$ turbulence model of Wilcox ${ }^{7}$ while in the core flow a LES is performed. The switching between RANS and LES regime is performed automatically by suitable transition functions. ${ }^{8}$

TASCOM3D uses a central spatial discretization of $6^{\text {th }}$ order with a small amount of upwind discretization of $5^{\text {th }}$ order with Multi-Dimensional Limiting Process - Low Dissipation ${ }^{9}$ (MLP ${ }^{\text {ld }}$ ). In contrast to conventional Total Variation Diminishing (TVD) limiters, the MLP ${ }^{\mathrm{ld}}$ scheme uses additional informations from diagonal volumes to improve shock resolution and convergence. The calculation of the inviscid fluxes is based on the Advection Upstream Splitting Method ${ }^{+}$up $^{10}\left(\mathrm{AUSM}^{+}\right.$-up). The temporal discretization is done fully implicit by a Backward Differentiation Formulas (BDF) method of up to $3^{\text {rd }}$ order. ${ }^{11}$ All entries of the required Jacobian matrix are set up analytically. The time integration is realized by a dual time stepping method. The inner iteration is assumed to be converged when the global residuum has decreased by at least three orders of magnitude. To enhance convergence, an all-Mach number preconditioning ${ }^{12}$ is implemented. Finally the nonlinear system of equation is solved using a Lower-Upper Symmetric Gauß-Seidel (LU-SGS) method. ${ }^{13}$

The high pressures and cryogenic temperatures occurring in the BKC do not allow the use of the Ideal Gas Equation of State (IG-EOS) to complete the equation system. Instead, the real gas behavior is described by the Soave-Redlich-Kwong Equation of State ${ }^{14}$ (SRK-EOS). The thermodynamic properties of the gas mixture is described by the model of Huber and Hanley. ${ }^{15}$ Combustion is modeled with detailed Finite-Rate Chemistry (FRC) using the reaction mechanism of Ó Conaire. ${ }^{16}$ This mechanism includes 8 species and 20 reactions. The Turbulence/Chemistry Interaction (TCI) is modeled by a multivariate assumed Probability 


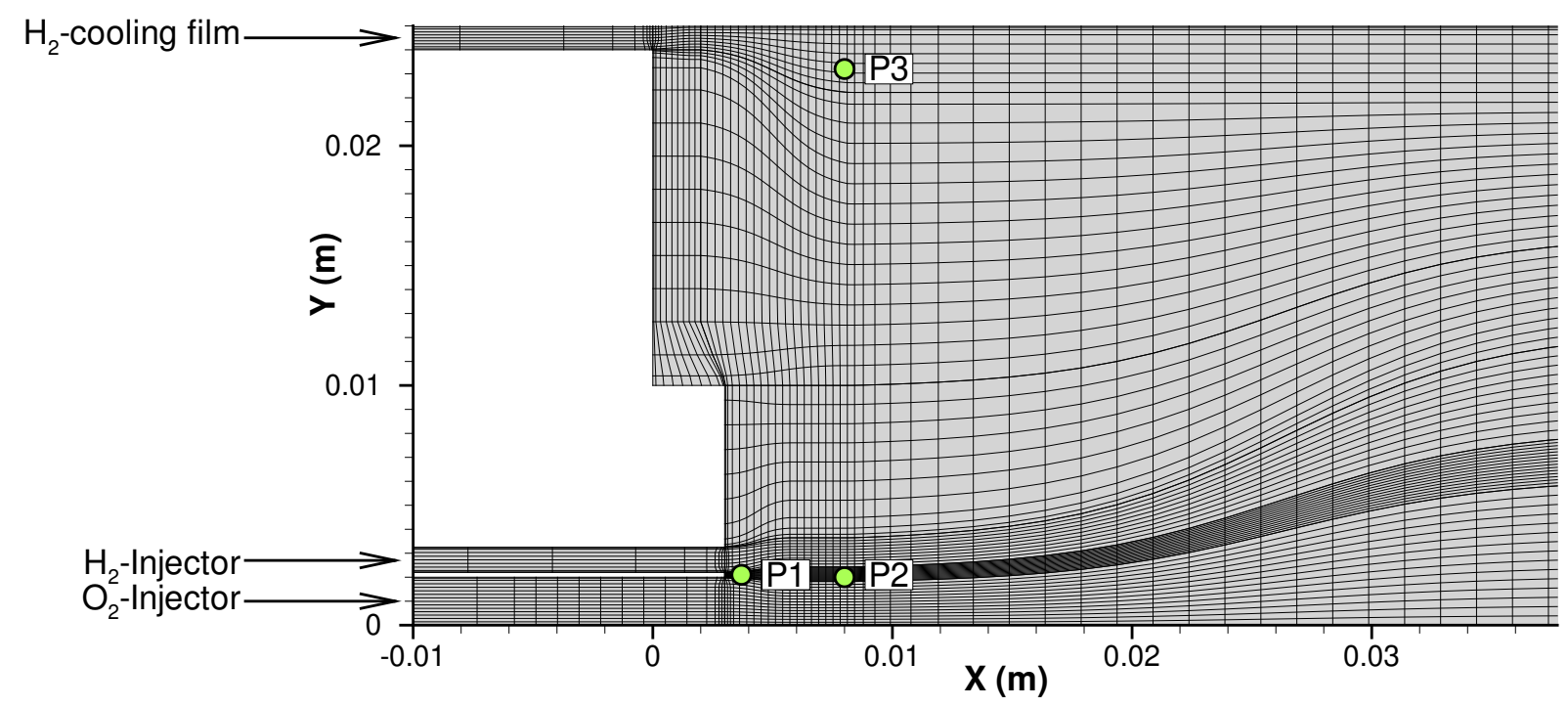

Figure 2. Injector geometry and computational grid close to the injector. For the sake of clarity, only every third volume is plotted in each direction. In addition the location of the points P1-P3 for the grid resolution study are shown.

Density Function ${ }^{17,18}$ (aPDF) method.

The numerical solver TASCOM3D uses structured grids. On one hand, this makes the code efficient and the high order discretization straightforward to implement. On the other hand, however, the grid generation becomes complicated for complex geometries. The three-dimensional grid of the BKC is meshed by 10.1 million volumes. It is a compromise between a good grid resolution and computational effort. The area from which the strongest effects on the flow field originate was expected to be the near injector region. For this reason, the grid has been strongly refined there. Likewise, considerable grid refinement is needed towards the walls to resolve the boundary layers and shear layers correctly. For the boundary layers of the BKC a dimensionless wall distance in the order of $y^{+} \leq 10$ is achieved. An extract from the grid in the near injector region is shown in Fig. 2 (every third volume in both directions for the sake of clarity). To ensure a fully developed turbulent inflow, the injectors are resolved up to $x=-0.05 \mathrm{~m}$. In order to reduce the computational effort, the grid gets coarser downstream of the injectors.

\section{Results and discussion}

\section{A. Analysis of grid resolution}

To check the quality of a LES grid, many different criteria are commonly used. Here, the amount of resolved turbulent kinetic energy and two-point correlations are applied. According to Pope ${ }^{19}$ a good LES resolution is achieved if more then $80 \%$ of the total turbulent kinetic energy is resolved. A corresponding parameter $\gamma$ is calculated by ${ }^{20}$

$$
\gamma=\frac{k_{\text {res }}}{k+k_{\text {res }}}, \quad k_{\text {res }}=\frac{1}{2}\left\langle u_{i}^{\prime} u_{i}^{\prime}\right\rangle,
$$

where the modeled turbulent kinetic energy $k$ is taken directly from the subgrid turbulence model.

In contrast, Davidson ${ }^{20,21}$ favours two-point correlations to determine the LES quality of the grid. The two-point correlation of the axial velocity $u$ is calculated as follows

$$
B_{u}(\hat{x})=\frac{\left\langle u^{\prime}(x) u^{\prime}(x-\hat{x})\right\rangle}{\left\langle u^{\prime}(x) u^{\prime}(x)\right\rangle} .
$$

According to Davidson a good grid resolution is achieved when the decrease of $B_{u}(\hat{x})$ for $\hat{x} \rightarrow \infty$ expands over more then ten cells. ${ }^{21}$

A corresponding analysis has been performed for six points in the combustor which are given in Table 2. The points P1-P3 are located in the highly resolved region close to the injectors: In the shear layer between 
Table 2. Amount of resolved turbulent kinetic energy at six different locations.

\begin{tabular}{ccccc}
\hline point & $x(\mathbf{m})$ & $y(\mathbf{m})$ & $z(\mathbf{m})$ & $\gamma(\mathbf{\%})$ \\
\hline P1 & 0.0037 & 0.0021 & 0.0 & 11.90 \\
P2 & 0.0080 & 0.0021 & 0.0 & 80.53 \\
P3 & 0.0080 & 0.0232 & 0.0 & 93.43 \\
P4 & 0.2003 & 0.0000 & 0.0 & 96.01 \\
P5 & 0.2003 & 0.0125 & 0.0 & 96.41 \\
P6 & 0.2003 & 0.0234 & 0.0 & 95.75 \\
\hline
\end{tabular}

oxygen and hydrogen (P1, P2) and in the shear layer of the $\mathrm{H}_{2}$ cooling flow (P3, see Figs. 2 and 3 ). The other three points are further downstream at an identical $x$-position at the symmetry axis, at half the radius and in the transition region between LES and RANS, respectively (see Fig. 3).

The fraction $\gamma$ of resolved turbulent kinetic energy is also given in Table 2. Despite the high grid resolution at point P1 less turbulent kinetic energy is resolved than at all other points. This is due to the switching from URANS to LES. P1 is located in the shear layer directly downstream of the oxygen and hydrogen injectors. The boundary layers of both injector tubes are calculated by URANS, where the turbulent kinetic energy is completely modeled. By convective transport, $k$ passes downstream to P1. Although the point P1 is already in LES regime, the high value of $k$ from the boundary layers are not be converted into resolved fluctuations due to the short distance between the upstream URANS regime and P1. At P2, which is somewhat further downstream, fluctuations have already developed and the flow is basically in the LES regime. As a result, more than $80 \%$ of then turbulent kinetic energy is resolved in P2 and the criterion of Pope ${ }^{19}$ is met.

Due to the results concerning the resolved turbulent energy at P1 no meaningful two-point correlation are expected at this point. For all other points from Table 2 two-point correlations are calculated for the axial velocity in $i$-coordinate direction, which basically corresponds to the x-direction. The two-point correlation of these points are shown in Fig. 4. From these figures it can be concluded that the resolution of the LES is good, since the decrease of the correlation extends over more then ten cells. ${ }^{21}$

The strong grid refinement close to the injectors as well as the finest turbulent structures found in this regime indicates an acceptable grid resolution. Also the rear part of the combustion chamber can be assumed to be reasonable resolved although the mesh is significantly coarsened here, because there is a high amount of resolved turbulent kinetic energy and good results from the two-point correlations.

\section{B. Comparison with experiment}

As the nozzle is included in the compressible flow simulation, the combustion chamber pressure $P_{C C}$ is part of the solution and is not given as a boundary condition. Therefore, the calculated combustion chamber pressure is a first indication of the quality of the simulation. In the experiment, a mean pressure of $P_{C C}=59.4$ bar ${ }^{3}$ is obtained. The time averaged pressure from the iDDES is calculated to $P_{C C}=60.9$ bar. Thus, the combustion chamber pressure is reproduced with an accuracy of $\sim 2.5 \%$.

For a further quantitative comparison a Fast Fourier Transformation (FFT) analysis of the pressure signal

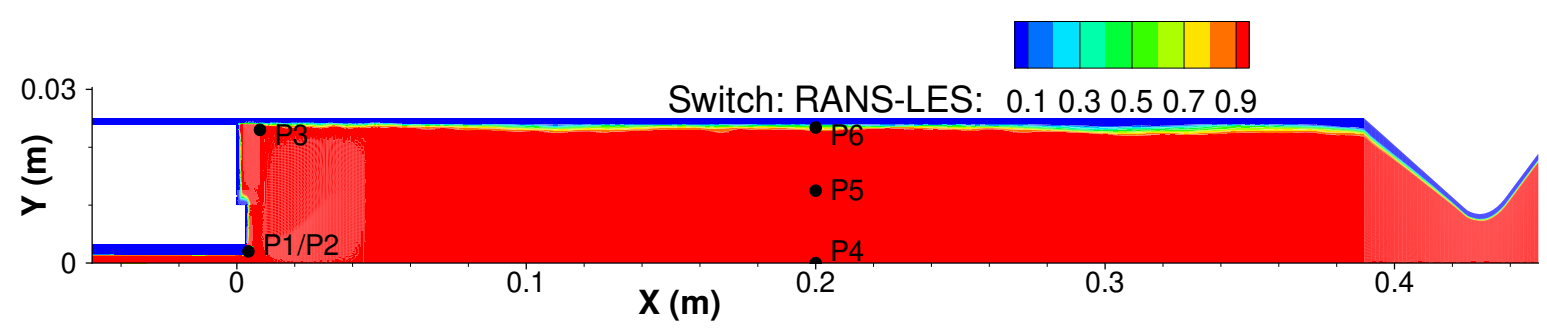

Figure 3. RANS (blue) and LES (red) regime of the iDDES. P1 to P6 indicate points for which statistics are calculated. 

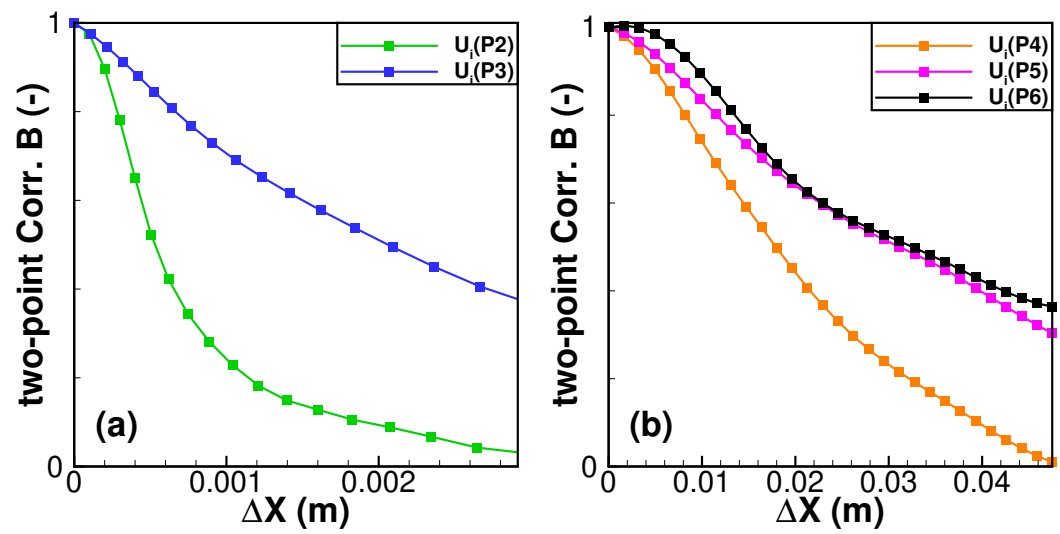

Figure 4. Two-point correlation for the different locations given in Table 2. Each symbol represent a grid cell.

is performed. In Fig. 5 the power spectral density (PSD) is plotted over the frequency for the experiment and the simulation. Smith et al. ${ }^{3}$ found the first longitudinal (1L) mode at $\sim 1800 \mathrm{~Hz}$. The simulation also resolves this resonant frequency but somewhat underestimates the amplitude. Because this is the fundamental mode of a standing longitudinal wave, the pressure bellies are located directly at the faceplate and in the nozzle throat. Thus, this pressure fluctuation can be observed at the monitor point MP1 (near the faceplate, see Fig. 1) but not at MP2, which is located in the middle of the combustion chamber. The second longitudinal (2L) mode measured and simulated has a frequency of $\sim 4000 \mathrm{~Hz}$. Now both MP1 and MP2 have a pressure belly and the mode is observed at both monitor points. Higher modes with significantly lower amplitudes are hardly visible in the simulation. The source of the strong experimentally observed pressure fluctuations at higher frequencies could not be clarified beyond doubt. It is possible that these are interactions with the pressure transducer ${ }^{3}$ and therefore are not seen in the simulation.

For a qualitative comparison of experiment and simulation shadowgraphs recorded in the area of optical access are available. The intensity of the experimentally recorded shadowgraphs depends on the distribution of the refractive index in the flow field. ${ }^{22}$ Since the refractive index depends on density, the intensity of the numerical shadowgraph $I_{C F D}$ is simplify calculated from the second derivative of the density ${ }^{23}$

$$
I_{C F D}=\left|\frac{\partial^{2} \rho}{\partial x_{i}^{2}}\right|
$$

In the experiment high density gradients cause dark regions in the shadowgraph while in the simulation high density gradients results in high values of $I_{C F D}$. For a good comparison, the color scale of the simulated shadowgraphs is chosen to tend towards black for high $I_{C F D}$ values, too. Fig. 6 shows an instant shadowgraph

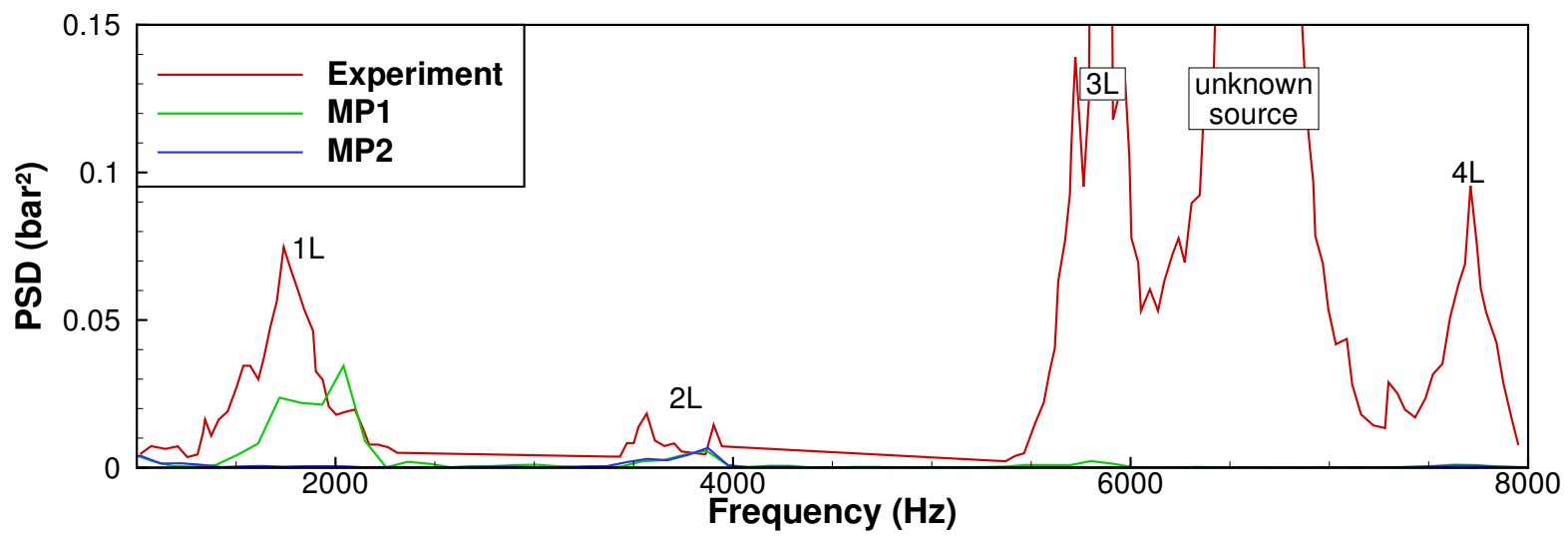

Figure 5. Comparison of the frequency spectrum from the experiment by Smith et al. ${ }^{3}$ with the simulation. 


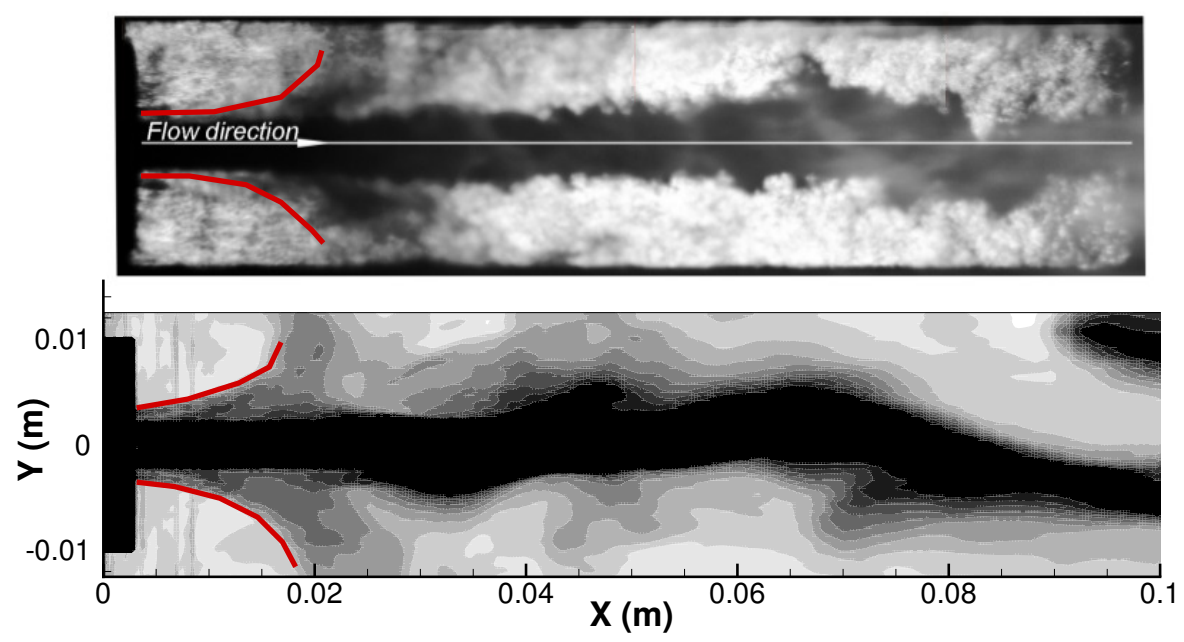

Figure 6. Comparison of experimentally determined (top) and simulated (bottom) shadowgraphs. Experiment by Smith et al. ${ }^{3}$

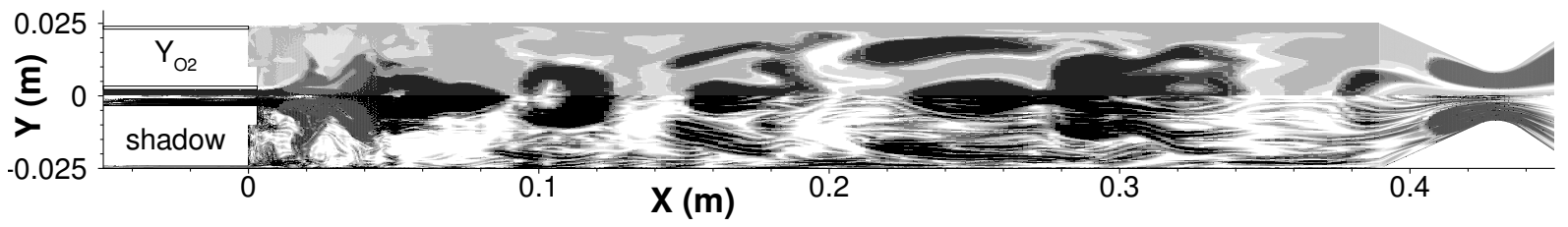

Figure 7. Comparison of a shadowgraph (bottom) and the corresponding $\mathrm{O}_{2}$ mass fraction distribution (top) obtained from the simulation.

of the experiment (top) compared to a simulated shadowgraph (bottom). A line of sight integration was performed for the simulation to make the pictures comparable. The overall agreement is good. Despite line of sight pictures are shown, small turbulent structures are visible in the experiment. This is in contrast to the line of sight CFD-result, where small structures are blurred. An explanation for this discrepancy could be that in the experiment the shadow image is not dominated exclusively by the density gradients but also by other phenomena such as the optical density of the cryogenic oxygen, which should be considered. However, in both experiment and simulation it can be clearly seen, how the $\mathrm{H}_{2}$-jet expands in radial direction (red lines at $x \approx 0.02 \mathrm{~m}$ ). Likewise, the length of the nearly undisturbed $\mathrm{O}_{2}$-jet is pretty well reproduced by the simulation.

The black regions (low intensity) along the axis of the shadowgraph in the experiment are interpreted as the oxygen core. ${ }^{4}$ A comparison of the shadowgraph from the simulation with the corresponding calculated $\mathrm{O}_{2}$ mass fraction distribution for the same instant of time in the $z=0$ plane is given in Fig. 7. Close to the axis, Fig. 7 shows a good agreement between the shadowgraph and the $\mathrm{O}_{2}$ mass fraction distribution. Here the shadowgraph is significantly influenced by the density gradients of the heating oxygen. On the other hand the shadowgraph exhibits much more structures in the vicinity of walls compared to the $\mathrm{O}_{2}$-image. Here, the shadowgraph is influenced by gradients between the high temperature regions of combustion and the cooling film. Nevertheless, the oxygen core length may be well estimated from density gradients.

\section{Flow field analysis and discussion}

The flow field in the near injector region is dominated by a number of vortices caused by the injected fluids, see Fig. 8. These vortices are, among other things, responsible for flame stabilisation. Fig. 8b shows in detail vortex 1 in the shearlayer between fuel and oxidizer. It can be seen that only oxygen is recirculated. This recirculation is very stable and nearly time independent. The fact that only oxygen is recirculated can be explained by the extreme expansion of the oxygen which is injected cryogenically with a density of about 


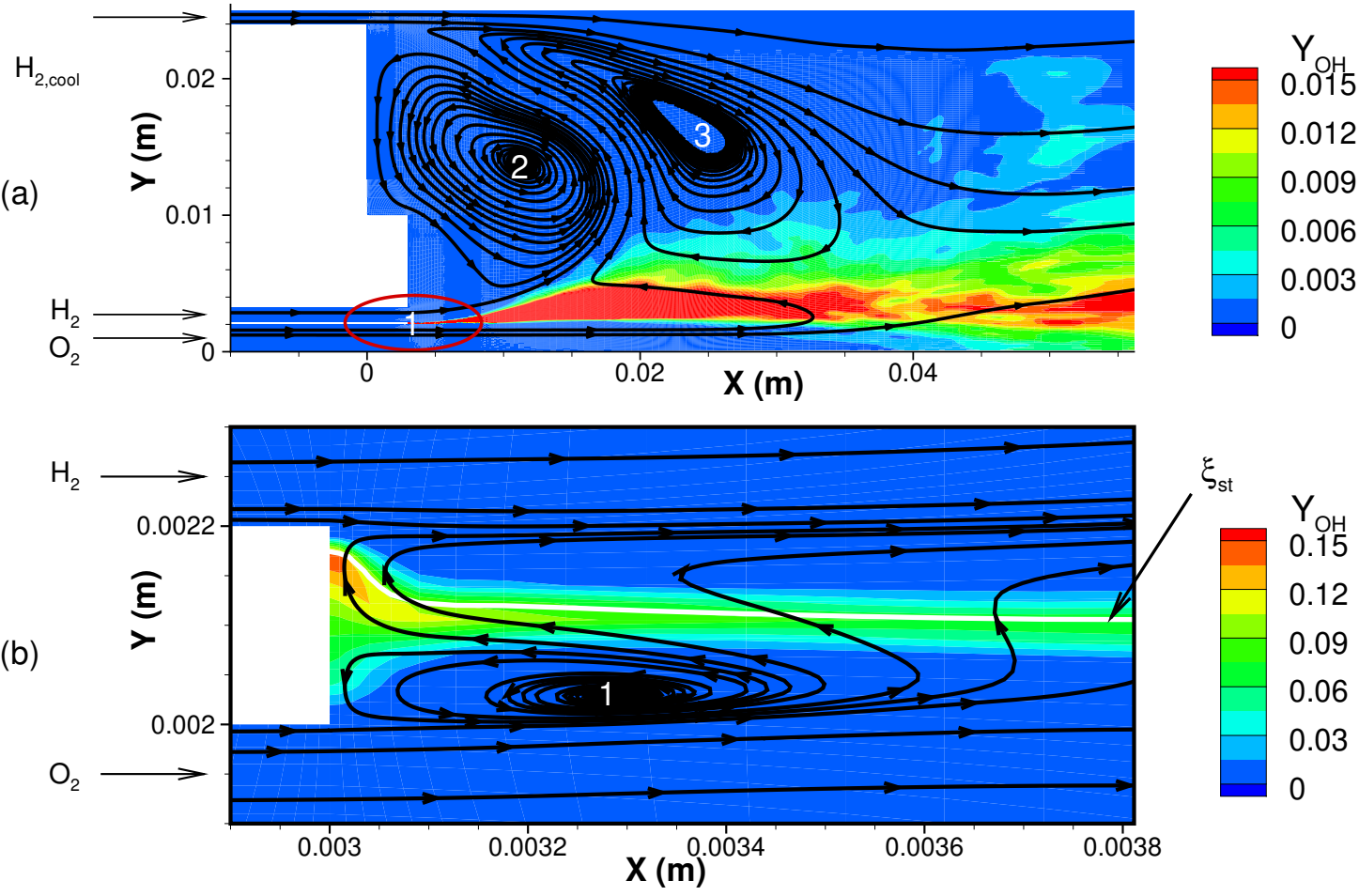

Figure 8. Recirculation zones close to the injectors: (a) first combustor part, (b) region directly downstream of the injector tubes. Contour plot shows $\mathrm{OH}$ mass fraction. Isoline of the stoichiometric mixture fraction $\xi_{s t}$ (white line).

$1050 \mathrm{~kg} / \mathrm{m}^{3}$. An increase in temperature of $100 \mathrm{~K}$ decreases the density by factor 7 . This forces spreading in radial direction (at $x \approx 0.0035 \mathrm{~m}$ ) and subsequently the recirculation of the oxidizer. This strong expansions prevents hydrogen from recirculating.

Fig. 8b also shows where combustion takes place due to increased $\mathrm{OH}$ concentration (colors in Fig. 8). The flame stabilizes directly at the injector post, where upstream transported oxygen and the entering hydrogen come together. The white isoline in Fig. 8 indicates the stoichiometric mixture fraction. The mixture fraction of the hydrogen-oxygen combustion is determined based on ${ }^{24}$ by

$$
\xi=Y_{\mathrm{H}_{2}}+\frac{1}{9} Y_{\mathrm{H}_{2} \mathrm{O}}
$$

where $Y$ is the mass fraction of hydrogen and water, respectively. For fast chemistry it can be assumed, that when there is stoichiometry, only products exist. This results in the stoichiometric mixture fraction $\xi_{s t}=\frac{1}{9}$ for the hydrogen-oxygen combustion. The recirculation of oxygen caused by vortex 1 and additionally the strong and fast energy release by the combustion of hydrogen and oxygen at high pressures prevents the flame from lifting off.

Two further vortices, which significantly influence the flow of the combustion chamber, are shown in Fig. 8a. In contrast to vortex 1 these vortices fluctuate strongly in time and space. The counter-rotating vortices are caused by the injected hydrogen at the center and the cooling flow at the combustion chamber wall. The extent and position of the vortices depend on the hydrogen injector conditions in interaction with the expanding oxygen jet.

In the combustor a wrinkled flame front is obtained as can be seen in Fig. 9, where the temperature field is shown. It can be seen from this figure that cold oxygen pockets are formed e.g. at $x \approx 0.28 \mathrm{~m}$. These are even better recognizable in the shadowgraph (Fig. 7). Schmitt ${ }^{25}$ found that such pockets are very stable and resilient to destruction by turbulence. This results in unburned oxygen leaving the combustion chamber despite fuel rich conditions. This is also observed in the experiment. ${ }^{4}$ 


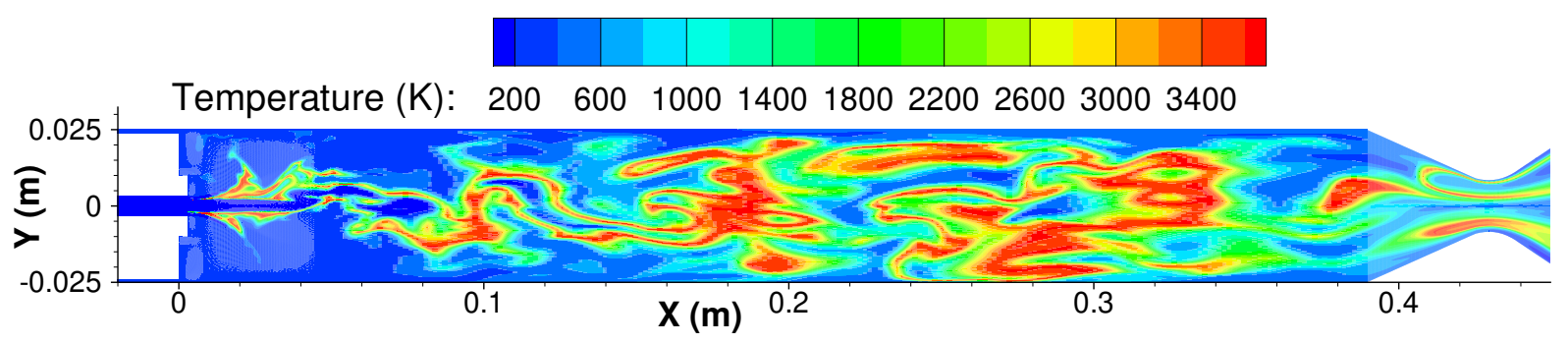

Figure 9. Instantaneous temperature field of the BKC in the $z=0$ slice
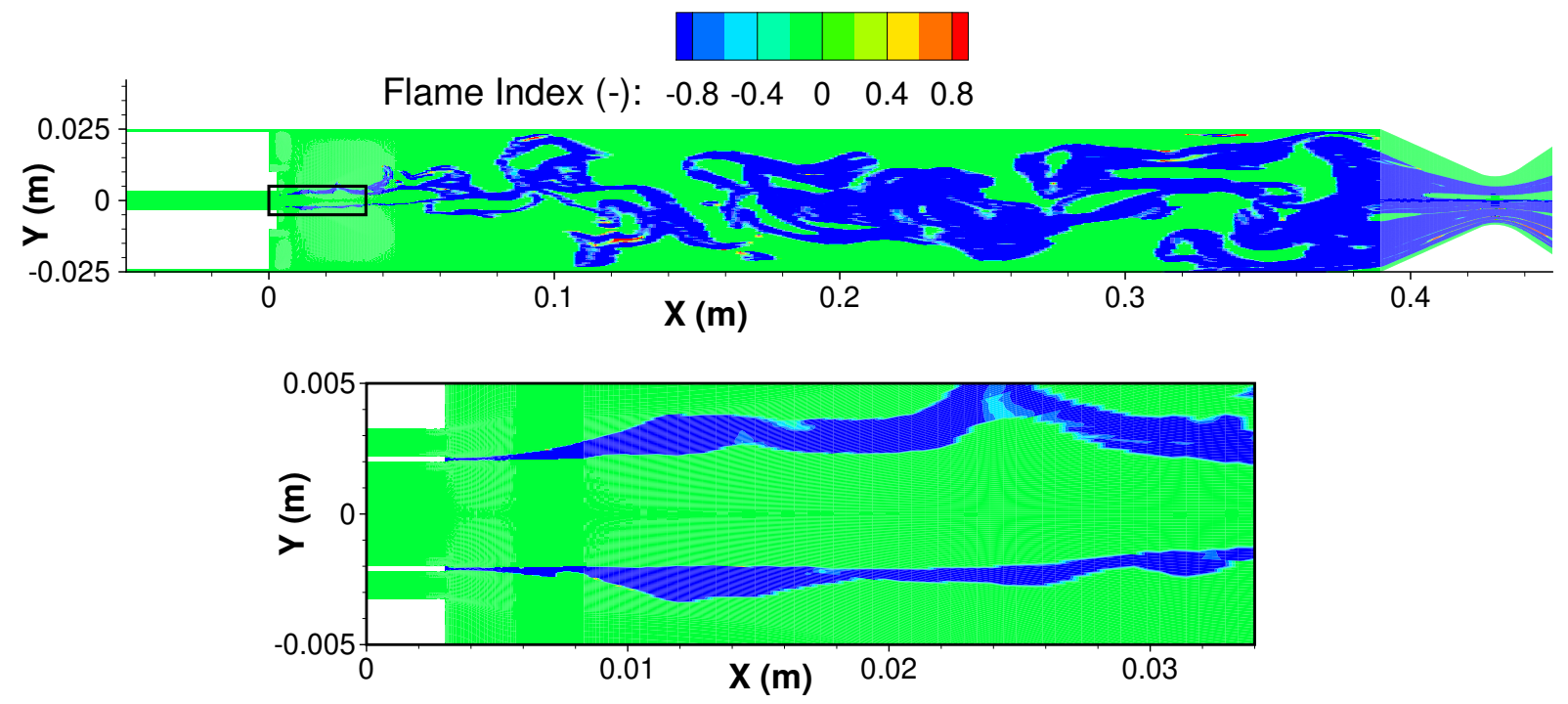

Figure 10. Flame index in the $z=0$ slice. Negative values show non premixed (diffusion) flames.

In order to investigate the combustion regime (premixed, non-premixed) in more detail, a flame index ${ }^{26,27}$

$$
F I= \begin{cases}\frac{\nabla Y_{\mathrm{H}_{2}} \cdot \nabla Y_{\mathrm{O}_{2}}}{\left|\nabla Y_{\mathrm{H}_{2}}\right|\left|\nabla Y_{\mathrm{O}_{2}}\right|}, & \text { if } Y_{\mathrm{OH}}>0.1 \% Y_{\mathrm{OH}, \max } \\ 0, & \text { otherwise }\end{cases}
$$

is calculated based on fuel and oxidizer gradients. This parameter only takes the resolved structures into account and neglects subgrid effects. Negative flame index values indicate non-premixed (diffusion) flames while positive values indicate premixed conditions. The minimum value of $\mathrm{OH}$ concentration is used here as an indicator for the flame position. The instantaneous flame index $F I$ for the BKC is plotted in Fig.10 in the $z=0$ plane. It shows that the entire combustion takes place in a diffusion flame mode. Even at the injector where is strong recirculation a diffusion flame is obtained. This is due to the high reactivity of the hydrogen-oxygen combustion at high pressure.

\section{Conclusion}

The in-house code TASCOM3D is used to investigate the model rocket combustion chamber BKC numerically at supercritical conditions. The performed simulation is a full three dimensional iDDES. The behavior of the real gas is modeled by the SRK-EOS while combustion is modeled whit finite-rate chemistry including aPDF approach.

In the course of the analysis, a study on the grid quality has been performed. According to the calculated amount of resolved turbulent kinetic energy and analysed two-point correlations the grid resolution is found 
at least as acceptable for a LES.

Results show good agreement with the experiment. The mean combustion chamber pressure is predicted accurately. Moreover, combustion chamber instabilities are resolved in accordance with the experiment. The frequency of this instabilities is determined accurately, while the amplitudes are somewhat underestimated. These combustion instabilities could not be resolved in a previous 2D-URANS simulation. Furthermore, shadow images calculated from the simulation are compared with the experiment. Good agreement is found here as well. The deflection of the hydrogen jet trough the expanding oxygen jet as well as the length of the undisturbed oxygen core could be reproduced by the simulation. Finally, the significant vortex structures and their influence on the combustion have been investigated.

\section{Acknowledgement}

The present work was conducted in the framework of the German Aerospace Center (DLR) project TAUROS (TAU for Rocket Thrust Chamber Simulation) focusing on the qualification and advancement of the DLR flow solver TAU for liquid rocket thrust chamber applications. The financial support of the DLR Space Research Programmatic is highly appreciated.

All simulations were performed on the Cray XC40 (hazelhen) cluster at the High Performance Computing Center Stuttgart (HLRS) under the grant number scrcomb. The authors wish to thank for the computing time and the technical support.

\section{References}

${ }^{1}$ Culick, F. E. and Yang, V., "Overview of combustion instabilities in liquid-propellant rocket engines," Liquid Rocket Engine Combustion Instability, Vol. 169, 1995, pp. 3-37.

${ }^{2}$ Simsont, Y. H. and Gerlinger, P., "High Order Numerical Simulation of the Thermal Load on a Lobed Strut Injector for Scramjet Applications," International Journal for Numerical Methods in Fluids, Vol. 82, No. 7, feb 2016, pp. 417-436.

${ }^{3}$ Smith, J., Klimenko, D., Clauss, W., and Mayer, W., "Supercritical LOX/hydrogen rocket combustion investigations using optical diagnostics," 38th AIAA/ASME/SAE/ASEE Joint Propulsion Conference E3 Exhibit, 2002, pp. 4033.

${ }^{4}$ Suslov, D., Hardi, J., Knapp, B., and Oschwald, M., "Hot-fire testing of liquid oxygen/hydrogen single coaxial injector at high-pressure conditions with optical diagnostics," 2015.

${ }^{5}$ Seidl, M., Numerische Simulation von Raketenbrennkammern mit transkritischer Einspritzung, Ph.D. thesis, Universität Stuttgart, 2018.

${ }^{6}$ Schäfer, K., Zimmermann, H., and Kruhsel, G., "Altitude Simulation Bench for VINCI Engine," 39th AIAA/ASME/SAE/ASEE Joint Propulsion Conference and Exhibit, American Institute of Aeronautics and Astronautics, jul 2003. 2838.

${ }^{7}$ Wilcox, D. C., "Formulation of the k- $\omega$ Turbulence Model Revisited," AIAA Journal, Vol. 46, No. 11, nov 2008, pp. $2823-$

${ }^{8}$ Shur, M. L., Spalart, P. R., Strelets, M. K., and Travin, A. K., "A hybrid RANS-LES approach with delayed-DES and wall-modelled LES capabilities," International Journal of Heat and Fluid Flow, Vol. 29, No. 6, 2008, pp. 1638-1649.

${ }^{9}$ Gerlinger, P., "Multi-dimensional limiting for high-order schemes including turbulence and combustion," Journal of Computational Physics, Vol. 231, No. 5, mar 2012, pp. 2199-2228.

${ }^{10}$ Liou, M.-S., "A sequel to AUSM, Part II: AUSM+-up for all speeds," Journal of Computational Physics, Vol. 214, No. 1, may 2006, pp. 137-170.

${ }^{11}$ Gear, C. W., Numerical Initial Value Problems in Ordinary Differential Equations, Prentice Hall, 1971.

${ }^{12}$ Yang, V., "Liquid-propellant rocket engine injector dynamics and combustion processes at supercritical conditions," Tech. rep., Pennsylvania State University Park Dept of Mechanical Engineering, 2004.

${ }^{13}$ Stoll, P., Entwicklung eines parallelen Mehrgitterverfahrens zur Simulation der Verbrennung in kompressiblen und inkompressiblen Strömungen, Ph.D. thesis, Universität Stuttgart, 2001.

${ }^{14}$ Soave, G., "Equilibrium constants from a modified Redlich-Kwong equation of state," Chemical Engineering Science, Vol. 27, 1972, pp. 1197-1203.

${ }^{15}$ Huber, M. and Hanley, H., "The corresponding-states principle: dense fluids," Transport properties of fluids, Vol. 285, 1996.

${ }^{16}$ Ó Conaire, M., Curran, H. J., Simmie, J. M., Pitz, W. J., and Westbrook, C. K., "A comprehensive modeling study of hydrogen oxidation," International Journal of Chemical Kinetics, Vol. 36, No. 11, 2004, pp. 603-622.

${ }^{17}$ Baurle, R. A., Alexopoulos, G. A., and Hassan, H. A., "Assumed joint probability density function approach for supersonic turbulent combustion," Journal of Propulsion and Power, Vol. 10, No. 4, jul 1994, pp. 473-484.

${ }^{18}$ Gerlinger, P., Numerische Verbrennungssimulation, Springer Berlin Heidelberg, 2005.

${ }^{19}$ Pope, S. B., "Ten questions concerning the large-eddy simulation of turbulent flows," New Journal of Physics, Vol. 6, mar 2004, pp. 35-35.

${ }^{20}$ Davidson, L., "Large Eddy Simulations: How to evaluate resolution," International Journal of Heat and Fluid Flow, Vol. 30, No. 5, oct 2009, pp. 1016-1025. 
${ }^{21}$ Davidson, L., "How to estimate the resolution of an LES of recirculating flow," Quality and Reliability of Large-Eddy Simulations II, Springer Netherlands, 2011, pp. 269-286.

${ }^{22}$ Settles, G. S., "Historical Background," Schlieren and Shadowgraph Techniques, Springer Berlin Heidelberg, 2001, pp. $1-24$.

${ }^{23}$ Schöpf, W., Patterson, J., and Brooker, A., "Evaluation of the shadowgraph method for the convective flow in a sideheated cavity," Experiments in fluids, Vol. 21, No. 5, 1996, pp. 331-340.

${ }^{24}$ Warnatz, J., Maas, U., Dibble, R. W., and Maas, U., Verbrennung, Springer-Verlag GmbH, 2001.

${ }^{25}$ Schmitt, T., Selle, L., Ruiz, A., and Cuenot, B., "Large-Eddy Simulation of Supercritical-Pressure Round Jets," AIAA Journal, Vol. 48, No. 9, sep 2010, pp. 2133-2144.

${ }^{26}$ Yamashita, H., Shimada, M., and Takeno, T., "A numerical study on flame stability at the transition point of jet diffusion flames," Symposium (International) on Combustion, Vol. 26, No. 1, jan 1996, pp. 27-34.

${ }^{27}$ Domingo, P., Vervisch, L., and Bray, K., "Partially premixed flamelets in LES of nonpremixed turbulent combustion," Combustion Theory and Modelling, Vol. 6, No. 4, dec 2002, pp. 529-551.

11 of 11 